\title{
Analysis of Competition between CPs Fronting Advertisers
}

\author{
Hamid Garmani ${ }^{1}$, Mohamed Baslam ${ }^{2}$ and Mostafa Jourhmane ${ }^{3}$ \\ 1,2,3 Information Processing and Decision Support Laboratory, Faculty of Sciences \\ and Technics, Sultan Moulay Slimane University, Beni Mellal, Morocco \\ 1'garmani.hamid@gmail.com, ${ }^{2}$ baslam.med@gmail.com, ${ }^{3}$ jourhman@hotmail.com
}

\begin{abstract}
This paper studies economic utilities, quality of service $(Q o S)$ and quality of content $(Q o C)$ in a network market. We propose new models that involve content Providers, end-users and advertisers. We consider a scenario where a content Provider $(C P)$ serves end-users that choose digital content. End-users have free access to each chosen $C P$; the $C P$ may have an advertisement revenue model (charging advertisers). We formulate the interactions between CPs as a non-cooperative game. In turn, the subscribers' demand for the content of a CP depends not only on their strategies, but also upon those proposed by all of its competitors. Through rigorous mathematical analysis, we prove existence and uniqueness of the Nash equilibrium. An iterative and distributed algorithm based on best response is proposed to achieve the equilibrium point. Finally, extensive simulations show convergence of a proposed scheme to the Nash equilibrium and give some insights on how the game parameters may vary the CPs strategies. This paper shows through economic analysis that the advertisers motivate the CP to invest on its contents.
\end{abstract}

Keywords: CP, advertisers, Game Theory, Nash Equilibrium, QoS, QoC

\section{Introduction}

The last years have seen much public debate and legislation initiatives concerning access to the global Internet. The Internet ecosystem is classically described as an interconnection of Autonomous Systems (ASs) [1], exchanging traffic through peering (free of charge) contracts or customer-provider contracts. The ASs have been classified by [2] into five categories: Large Transit Providers, Small Transit Providers, Access/Hosting Providers, Enterprise Customers, and Content Providers.

From an end-user perspective, the current Internet pricing model involves two customer-content provider relationships: (i) Access fees, where end users pay a fee to access to content provided by the Content Providers (CP), based, in general, on flat offers. (ii) Access free, where end users have a free access to the Content Providers $(\mathrm{CP})$ and $\mathrm{CP}$ gets its revenue from advertisers.

Game theory has been widely used as a central tool for the design of future wireless and communication networks in recent years [3]. Game theory formulated interaction main incentive structures. It is a mathematical study of the theory and methods of the competitive nature of the phenomenon. Game theory considers the game to predict individual behavior and actual behavior, and to study their optimization strategy.

Game theory provides a natural framework for modeling selfish providers in telecommunication market [4-9]. In [10] the authors studied game problems involving two types of content providers: one that corresponds to independent content providers, and one that correspond to content providers that have exclusive agreements with Internet Service Providers (ISPs). In [11] the authors considered

Received (March 9, 2018), Review Result (May 10, 2018), Accepted (May 18, 2018) 
that, the CPs faced with the question of how to choose in what content to specialize. They showed that the problem of selecting the content type is equivalent to a congestion game. Several works have been studied CPs obtain revenue from end-users [12-16]. In this paper we study CP get their revenue from advertisers and offer free access to end-users for access to their content.

In this work, we consider internet supply chain with CPs, advertisers, and end-users. Thought, in practice, we often have CPs in competition for end-users and advertisers. The CP sets their credibility of content level according to the price paid by advertisers. End-users adapt their demand of content based on the CPs policies.

The main contribution of this paper are as follows:

- We present new features in the mathematical modeling that include CPs revenues.

- We include quality of service and quality of content in competition between CPs for end-users and advertisers at the demand markets.

- We modeling competition between the CPs based on two market parameters, quality of service and quality of content, where each CPs want to maximize their utility. We formulate the competitive problem between CPs as non-cooperative game.

- We analytically prove the existence and uniqueness of Nash equilibrium in non-cooperative game between $\mathrm{CPs}$, which means that there exists a stable state where all CPs do not have an incentive to change their strategies.

- We complement our analysis with numerical results that shows the impact of advertisers' investment on CPs policies.

The rest of this work is organized as follows. In Section 2 we describe the system model, content credibility, demand model and utility functions. In Section 3 we formulate a non-cooperative game, and proof the existence and uniqueness of Nash equilibrium solution. Then, we present numerical results in Section 4. In Section 5 Conclusion.

\section{System Model}

We consider an Internet ecosystem that consists of tree economic entities, namely the advertisers, the CPs, and an arbitrary number of users who can switch from one CP to another. CP provides its contents to the users using networks architecture provided by ISPs. Each CP sets two market parameters quality of service QoS and quality of content QoC. The CP usually has two revenue models, the user subscription and the advertisement from clicks of users. These two models, though sometimes coexisting with each other, in this work we study the obtain revenue from the advertisers. For the advertisement based model, the CP attracts users' clicks on online advertisements. The internet service provide (ISP) charges the CP to transmit their content with credibility to customers. Advertisers provide the revenue of content providers and put advertisement in content provided by the content providers. End user subscribes to content providers provide a better service.

Figure 1 represent a system with $\mathrm{M}$ advertisers, $\mathrm{N} \mathrm{CPs}$, and customer market. $p_{a_{i}}$ represents price of per attention guaranteed by $C P_{i}$. 


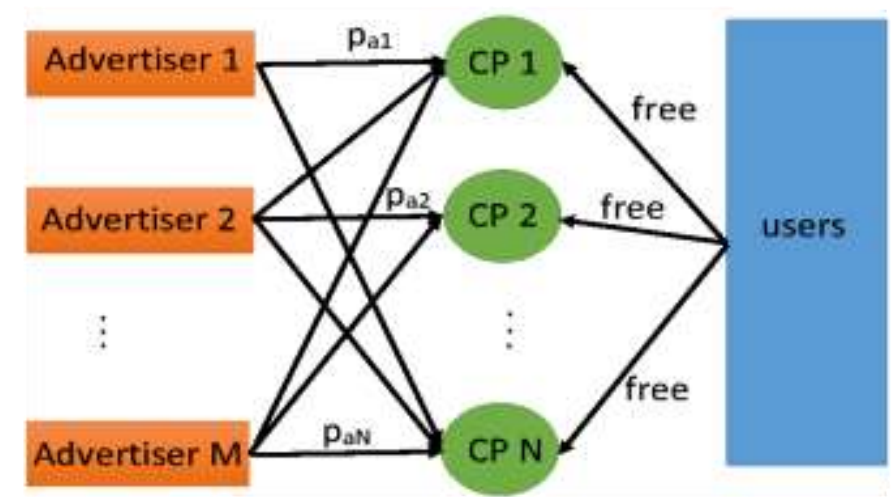

Figure 1. Money Flow in the Internet Market

\subsection{Content Credibility}

We assume that the credibility of content $c_{i}$ of $C P_{i}$ is a function of the quality of service $q_{s_{i}}$ and quality content $q_{c_{i}}$, which is written as follows:

$$
c_{i}=\lambda q_{s_{i}}+\mu q_{c_{i}}
$$

$\lambda$ and $\mu$ are two positive constants, which represent respectively the sensitivity of the credibility of content to QoS and QoC.

We define the QoS as the "expected delay", which is computed by the Kleinrock function (see [17]) as the reciprocal of the square root of delay:

$$
q_{s_{i}}=\frac{1}{\sqrt{\text { Delay }}}=\sqrt{b_{i}-D_{i}}
$$

$b_{i}$ is the amount of bandwidth required by $C P_{i}$.

The quality of content QoC can be specified for a specific domain of content, e.g., quality of video streaming.

\subsection{Demand Model}

\subsubsection{Subscription Model}

$D_{i}$ the average demand of all users for the content provided by $C P_{i}$, which depends on the credibility $c_{i}$ (see [18],[19]). This demand function depends also on credibility $c_{-i}$ set by the competitors. Eventually, $D_{i}$ is increasing w.r.t $c_{i}$ and decreasing w.r.t. $c_{n}, n \neq i$.

Then, the demand functions $D_{i}$ can be written as follows:

$$
D_{i}(c)=d_{i}+\beta_{i}^{i} c_{i}-\sum_{n, n \neq i}^{N} \beta_{i}^{n} c_{n}
$$

where $d_{i}$ is a positive constant used to insure non-negative demands over the feasible region. While $\beta_{i}^{n}$ are positive constants representing the sensitivity of $C P_{i}$ to credibility of $C P_{n}$. 
The Demand Function $D_{i}$ is written as follows:

$$
D_{i}\left(q_{s}, q_{c}\right)=d_{i}+\beta_{i}^{i}\left(\lambda q_{s_{i}}+\mu q_{c_{i}}\right)-\sum_{n=1, n \neq i}^{N} \beta_{i}^{n}\left(\lambda q_{s_{n}}+\mu q_{c_{n}}\right)
$$

Where

$$
\sum_{n=1}^{N} \beta_{i}^{n}=1, \quad i=1, \ldots, N
$$

\section{Assumption 1}

The credibility sensitivity $\beta$ verifies:

$$
\beta_{i}^{i} \geq \sum_{n, n \neq i} \beta_{i}^{n}, \forall i, n=1, \ldots, N
$$

Assumption 1 will be needed to ensure the uniqueness of the resulting equilibrium. The assumption 1 means that the influence of QoS (resp QoC) the CP on its demand is greater than the influence of the QoS (resp QoC) of its opponents on its demand.

\subsubsection{Advertisement Model}

In this paragraph, we describe the economic interaction between advertisers and the $C P_{i}$. There are $M$ advertisers, each advertiser interested in the $C P_{i}$ has a fixed budget $B_{i}$ in a given time interval (e.g., daily, weekly or monthly) and has a valuation $v_{i}$ to declare its maximum willingness to pay for each attention to $C P_{i}$. The valuation $v_{i}$ is a random variable in the range $\left[0, \overline{v_{i}}\right]$. Suppose that $v_{i}$ is characterized by probability density function $x\left(v_{i}\right)$ and cumulative distribution function (CDF) $\mathrm{X}(\mathrm{v})$. We assume that the valuations of all advertisers are independent and identically distributed. Let $p_{a_{i}}$ be the price of per attention charged by the $C P_{i}$. We denote by $D_{a_{i}}$ the demand of attentions from advertisers to the $C P_{i}$. Therefore, $D_{a_{i}}$ can be expressed as [20]:

$$
D_{a_{i}}=\frac{M B_{i}}{p_{a_{i}}} \operatorname{prob}\left(v_{i} \geq p_{a_{i}}\right)
$$

Where $\operatorname{prob}\left(v_{i} \geq p_{a_{i}}\right)=1-X\left(p_{a_{i}}\right)$ then:

$$
D_{a_{i}}=\frac{M B_{i}}{p_{a_{i}}}\left(1-X\left(p_{a_{i}}\right)\right)
$$

When $C P_{i}$ increase $p_{a_{i}}$, the attention of advertisers to $C P_{i}$ will decrease. However, the demand of attentions from advertisers to the $C P_{i}$, is upper bounded by the demand of users $C P_{i}$. Therefore, $D_{a_{i}}$ can expressed as [20]

$$
D_{a_{i}}=\min \left\{D_{i}, \frac{M B_{i}}{p_{a_{i}}}\left(1-X\left(p_{a_{i}}\right)\right)\right\}
$$


Suppose $v_{i}$ follows a uniform distribution in the range $\left[0, \overline{v_{i}}\right]$. Then, $X\left(p_{a_{i}}\right)=\frac{p_{a_{i}}}{\overline{v_{i}}}$ the demand becomes:

$$
D_{a_{i}}=\frac{M B_{i}}{p_{a_{i}}}\left(1-\frac{p_{a_{i}}}{\overline{v_{i}}}\right)
$$

To find the optimal price we follow [20], the optimal price $p^{a}$ is obtained when:

$$
D_{i}=\frac{M B_{i}}{p_{a_{i}}}\left(1-\frac{p_{a_{i}}}{\overline{v_{i}}}\right)
$$

Then, the optimal price of per attention,

$$
p_{a_{i}}=\frac{M B_{i} \overline{v_{i}}}{M B_{i}+D_{i} \overline{v_{i}}}
$$

\section{Utility}

Let $U_{i}$ be the profit function of $C P_{i}$, then it is given by:

$$
U_{i}=p_{a_{i}} D_{a_{i}}-\omega_{i} c_{i}-c_{i} p_{t} D_{i}
$$

where $p_{a_{i}} D_{a_{i}}$ the revenue of $C P_{i}$ from advertisers. $\omega_{i}$ is the cost to produce unit of credibility of content $c_{i} \cdot \omega_{i} c_{i}$ the cost to produce credibility of content $c_{i} \cdot p_{t}$ transmission fee. $c_{i} p_{t} D_{i}$ transmission fee of $C P_{i}$ when transmitting content with credibility $c_{i}$.

Submitting equation 1,11 in equation 12 , the $C P_{i}$ utility become:

$$
U_{i}=\frac{M B_{i} \overline{v_{i}} D_{i}}{M B_{i}+D_{i} \overline{v_{i}}}-\omega_{i} c_{i}-c_{i} p_{t} D_{i}
$$

\section{A Non-Cooperative Game Formulation}

Let $G_{1}=\left\lfloor\mathrm{H},\left\{Q_{s_{i}}, Q_{c_{i}}\right\},\left\{U_{i}().\right\}\right]$ denote the non-cooperative QoS QoC game (NQQG), where $\mathrm{H}=\{1, \ldots, \mathrm{N}\}$ is the index set identifying the CPs, $Q_{s_{i}}$ is the QoS strategy set of $C P_{i}, Q_{c_{i}}$ is the QoC strategy set of $C P_{i}$ and $U_{i}($.$) is the utility function. Let the QoS$ vector $\quad q_{s}=\left(q_{s_{1}}, \ldots, q_{s_{N}}\right)^{T} \in Q_{s}^{N}=Q_{s_{1}} \times Q_{s_{2}} \times \ldots \times Q_{s_{N}} \quad, \quad$ QoC vector $q_{c}=\left(q_{c_{1}}, \ldots, q_{c_{N}}\right)^{T} \in Q_{c}^{N}=Q_{c_{1}} \times Q_{c_{2}} \times \ldots \times Q_{c_{N}}$. The utility of $C P_{i}$ when it decides the strategy $q_{s_{i}}$ and $q_{c_{i}}$ is given in equation 13 . We assume that the strategy spaces $Q_{s_{i}}$ and $Q_{c_{i}}$ of each CPs are compact and convex sets with maximum and minimum constraints, for any given $C P_{i}$ we consider strategy spaces the closed intervals $Q_{s_{i}}=\left[\underline{q}_{s_{i}}, \bar{q}_{s_{i}}\right]$ and $Q_{c_{i}}=\left[\underline{q}_{c_{i}}, \bar{q}_{c_{i}}\right]$. 
In order to maximize their utilities, each $C P_{i}$ decides QoS $q_{s_{i}}$ and QoC $q_{c_{i}}$. Formally, the problem can be expressed as :

$$
\max _{q_{s_{i}} \in Q_{s_{i}}, q_{c_{i}} \in Q_{c_{i}}} U_{i}\left(q_{s}, q_{c}\right), \quad \forall i \in \mathrm{H} .
$$

In this section, we show that the non-cooperative game $G_{1}$ fulfills the properties of the Debreu-Glicksberg-Fan Theorem [21] and therefore admits a NE.

\subsection{Fixed QoS Game}

Considering some fixed QoS policy, a Nash equilibrium in QoC is formally defined as:

Definition $1 \mathrm{~A}$ QoC vector $q_{c}^{*}=\left(q_{c_{1}}^{*}, \ldots, q_{c_{N}}^{*}\right)$ is a Nash equilibrium of the NQQG game $G_{1}=\left[\mathrm{H},\left\{Q_{s_{i}}, Q_{c_{i}}\right\},\left\{U_{i}\left(q_{s}, q_{c}\right)\right\}\right]$ if:

$$
\forall\left(i, q_{c_{i}}\right) \in\left(\mathrm{H}, Q_{c_{i}}\right), U_{i}\left(q_{c_{i}}^{*}, q_{c_{-i}}^{*}\right) \geq U_{i}\left(q_{c_{i}}, q_{c_{-i}}^{*}\right)
$$

Theorem 1 A Nash equilibrium in terms of QoC for game $G_{1}=\left\lfloor\mathrm{H},\left\{Q_{s_{i}}, Q_{c_{i}}\right\}\left\{U_{i}\left(q_{s}, q_{c}\right)\right\}\right]$ exists and is unique.

Proof:

To prove existence, we note that each CPs strategy space $Q_{c_{i}}$ is defined by all QoCs in the closed interval bounded by the minimum and maximum QoCs. Thus, the joint strategy space $Q_{c}$ a nonempty, convex, and compact subset of the Euclidean space $R^{N}$. In addition, the utility functions are concave with respect to QoCs as can be seen from the second derivative test:

$$
\frac{\partial U_{i}}{\partial q_{c_{i}}}=\frac{\beta_{i}^{i} \mu M^{2} B_{i}^{2} \overline{v_{i}}}{\left(M B_{i}+D_{i} \overline{v_{i}}\right)^{2}}-\omega_{i} \mu-\mu p_{t} D_{i}-\beta_{i}^{i} \mu\left(\lambda q_{s_{i}}+\mu q_{c_{i}}\right) p_{t}
$$

Then,

$$
\frac{\partial^{2} U_{i}}{\partial q_{c_{i}}^{2}}=-\frac{2\left(\beta_{i}^{i}\right)^{2} \mu^{2} M^{2} B_{i}^{2}{\overline{v_{i}}}^{2}}{\left(M B_{i}+D_{i} \bar{v}_{i}\right)^{3}}-2 \beta_{i}^{i} \mu^{2} p_{t} \leq 0
$$

The second derivative of the utility function is negative, then the utility function is thus concave, which ensures existence of a Nash equilibrium.

We use the following proposition that holds for a concave game [21]: If a concave game satisfies the dominance solvability condition:

$$
-\frac{\partial^{2} U_{i}}{\partial q_{c_{i}}^{2}} \geq \sum_{n, n \neq i}\left|\frac{\partial^{2} U_{i}}{\partial q_{c_{i}} \partial q_{c_{n}}}\right|
$$

then the game $G_{1}$ admits a unique NE.

The mixed partial is written as:

$$
\frac{\partial^{2} U_{i}}{\partial q_{c_{i}} \partial q_{c_{n}}}=\frac{2 \beta_{i}^{i} \beta_{i}^{n} \mu^{2} M^{2} B_{i}^{2} \bar{v}_{i}^{2}}{\left(M B_{i}+D_{i} \bar{v}_{i}\right)^{3}}+\mu^{2} \beta_{i}^{n} p_{t}
$$

Then, 
$-\frac{\partial^{2} U_{i}}{\partial q_{c_{i}}^{2}}-\sum_{n, n \neq i}\left|\frac{\partial^{2} U_{i}}{\partial q_{c_{i}} \partial q_{c_{n}}}\right|=\frac{2 \beta_{i}^{i} \mu^{2} M^{2} B^{2} v^{2}}{\left(M B_{i}+D_{i} \bar{v}_{i}\right)^{3}}\left(\beta_{i}^{i}-\sum_{n, n \neq i} \beta_{i}^{n}\right)+\mu^{2} p_{t}\left(2 \beta_{i}^{i}-\sum_{n, n \neq i} \beta_{i}^{n}\right) \geq 0$

Thus, the Nash equilibrium point is unique.

\subsection{Fixed QoC Game}

Considering some fixed price and QoC policy, a Nash equilibrium in QoS is formally defined as:

Definition 2 A QoS vector $q_{s}^{*}=\left(q_{s_{1}}^{*}, \ldots, q_{s_{N}}^{*}\right)$ is a Nash equilibrium of the NQQG game $G_{1}=\left[\mathrm{H},\left\{Q_{s_{i}}, Q_{c_{i}}\right\},\left\{U_{i}\left(q_{c}, q_{s}\right)\right\}\right]$ if :

$$
\forall\left(i, q_{s_{i}}\right) \in\left(\mathrm{H}, Q_{s_{i}}\right), U_{i}\left(q_{s_{i}}^{*}, q_{s_{-i}}^{*}\right) \geq U_{i}\left(q_{s_{i}}, q_{s_{-i}}^{*}\right)
$$

Theorem 2 A Nash equilibrium in terms of QoS for game $G_{1}=\left\lfloor\mathrm{H},\left\{Q_{s_{i}}, Q_{c_{i}}\right\}\left\{U_{i}\left(q_{c}, q_{s}\right)\right\}\right]$ exists and is unique.

Proof:

To prove existence, we note that each CPs strategy space $Q_{s_{i}}$ is defined by all QoSs in the closed interval bounded by the minimum and maximum QoSs. Thus, the joint strategy space $Q_{s}$ a nonempty, convex, and compact subset of the Euclidean space $R^{N}$. In addition, the utility functions are concave with respect to QoSs as can be seen from the second derivative test:

$$
\frac{\partial U_{i}}{\partial q_{s_{i}}}=\frac{\beta_{i}^{i} \lambda M^{2} B_{i}^{2} \overline{v_{i}}}{\left(M B_{i}+D_{i} \overline{v_{i}}\right)^{2}}-\omega_{i} \lambda-\lambda p_{t} D_{i}-\beta_{i}^{i} \lambda\left(\lambda q_{s_{i}}+\mu q_{c_{i}}\right) p_{t}
$$

Then,

$$
\frac{\partial^{2} U_{i}}{\partial q_{s_{i}}^{2}}=-\frac{2\left(\beta_{i}^{i}\right)^{2} \lambda^{2} M^{2} B_{i}^{2}{\overline{v_{i}}}^{2}}{\left(M B_{i}+D_{i} \overline{v_{i}}\right)^{3}}-2 \beta_{i}^{i} \lambda^{2} p_{t} \leq 0
$$

The second derivative of the utility function is negative, then the utility function is thus concave, which ensures existence of a Nash equilibrium.

We use the following proposition that holds for a concave game [21]: If a concave game satisfies the dominance solvability condition:

$$
-\frac{\partial^{2} U_{i}}{\partial q_{s_{i}}^{2}} \geq \sum_{n, n \neq i}\left|\frac{\partial^{2} U_{i}}{\partial q_{s_{i}} \partial q_{s_{n}}}\right|
$$

then the game $G_{1}$ admits a unique NE.

The mixed partial is written as:

$$
\frac{\partial^{2} U_{i}}{\partial q_{s_{i}} \partial q_{s_{n}}}=\frac{2 \beta_{i}^{i} \beta_{i}^{n} \lambda^{2} M^{2} B_{i}^{2}{\overline{v_{i}}}^{2}}{\left(M B_{i}+D_{i} \overline{v_{i}}\right)^{3}}+\lambda^{2} \beta_{i}^{n} p_{t}
$$

Then,

$$
-\frac{\partial^{2} U_{i}}{\partial q_{s_{i}}^{2}}-\sum_{n, n \neq i}\left|\frac{\partial^{2} U_{i}}{\partial q_{s_{i}} \partial q_{s_{n}}}\right|=\frac{2 \beta_{i}^{i} \lambda^{2} M^{2} B_{i}^{2} \bar{v}_{i}^{2}}{\left(M B_{i}+D_{i} \overline{v_{i}}\right)^{3}}\left(\beta_{i}^{i}-\sum_{n, n \neq i} \beta_{i}^{n}\right)+\lambda^{2} p_{t}\left(2 \beta_{i}^{i}-\sum_{n, n \neq i} \beta_{i}^{n}\right) \geq 0
$$

Thus, the Nash equilibrium point is unique. 


\subsection{Learning Nash Equilibrium QoS, QoC}

The section mentioned above show clearly that the Nash equilibrium is unique. Now, we turn to develop a fully algorithms that converge quickly to Nash equilibrium. However, we assume that each CPs has a perfect information on strategies of its competitors. Each CPs fixes its desirable strategies in order to maximize its own profit. Then, each CPs can observe the policy taken by its competitors in previous rounds and input them in its decision process to update its policy. Then, it becomes natural to accept Nash equilibrium as the attracting point of the game. Therefore, the best response algorithm will converge a to unique equilibrium. The best response algorithm is summarized in algorithm 1 .

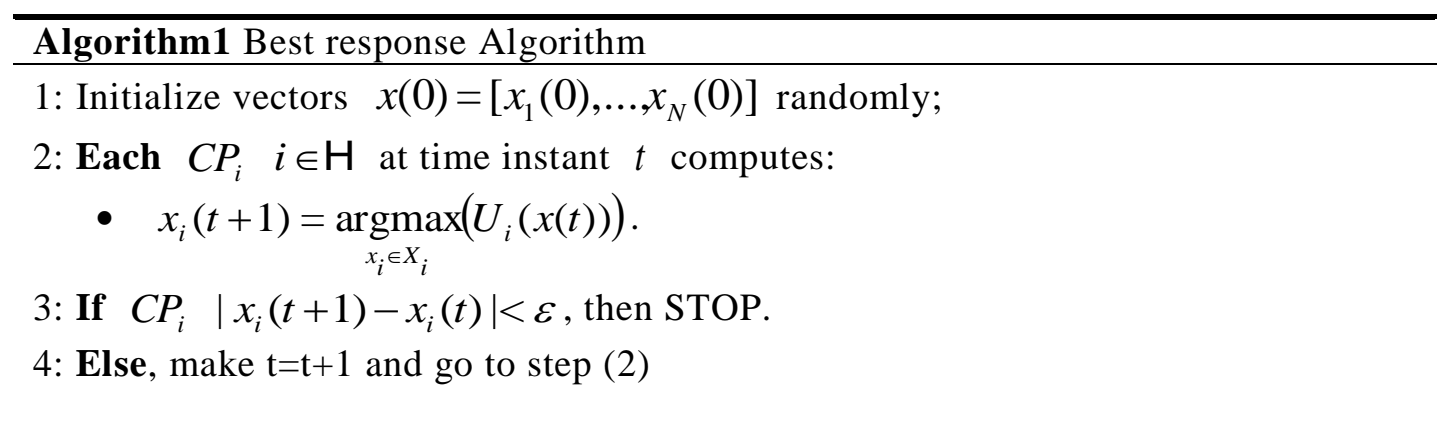

\section{Such as:}

- $\mathrm{x}$ denotes the vector $q_{s}$ and vector $q_{c}$.

- $X_{i}$ denotes the policy profile QoS or QoC.

\subsection{Price of Anarchy}

The price of anarchy is the ratio of the worst case objective function value of a Nash equilibrium and that of an optimal outcome. That measures how the efficiency of a system degrades due to selfish behavior of its agents. The price of anarchy [22] is a method to measure the inefficiency of equilibrium; it has been used to measure the inefficiency in congestion networks. In this case, each user of the network has a source and destination and they must pay a cost to travel from their source to their destination. In this case it is given by:

$$
P o A=\frac{\min _{p_{s}, p_{c}, q_{s}, k} W_{N E}\left(p_{s}, p_{c}, q_{s}, k\right)}{\max _{p_{s}, p_{c}, q_{s}, k} W\left(p_{s}, p_{c}, q_{s}, k\right)}
$$

where $W\left(p_{s}, p_{c}, q_{s}, k\right)=\sum_{j=1}^{M} U_{i}\left(p_{s}, p_{c}, q_{s}, k\right) \quad$ is $\quad$ a welfare function and $W_{N E}\left(p_{s}^{*}, p_{c}^{*}, q_{s}^{*}, k^{*}\right)=\sum_{j=1}^{M} U_{i}\left(p_{s}, p_{c}, q_{s}, k\right)$ is a sum of utilities of all actors at Nash Equilibrium.

\section{Numeric Analysis}

To clarify and show how to take advantage from our theoretical study, we suggest to studying numerically the market share game while considering the best response algorithm and expressions of the utility function of CPs. Hence, we consider a system with three CPs and 1500 advertisers. 


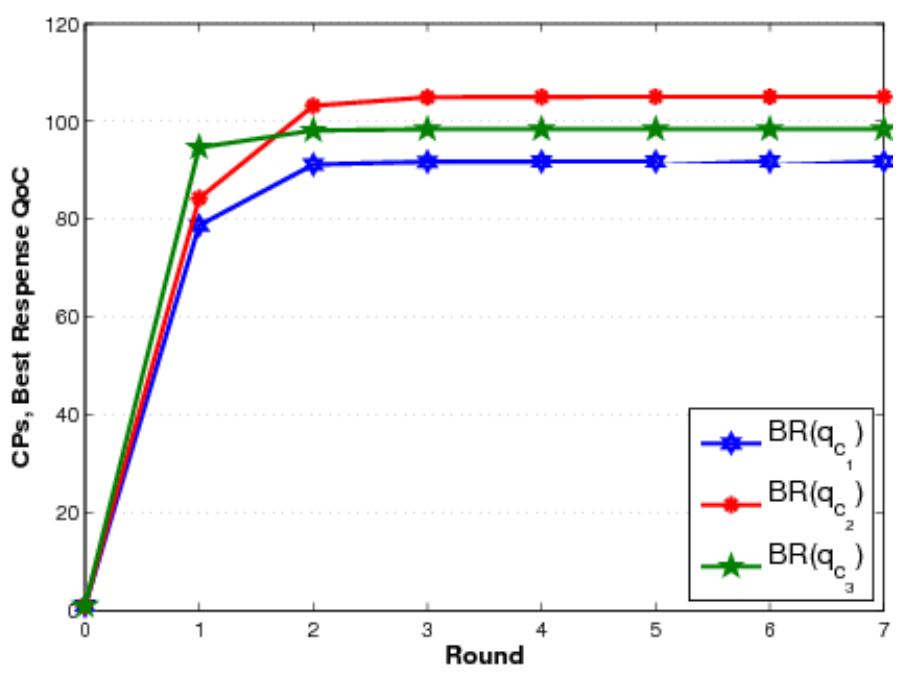

Figure 2. QoC Game: Convergence to the QoC Nash Equilibrium

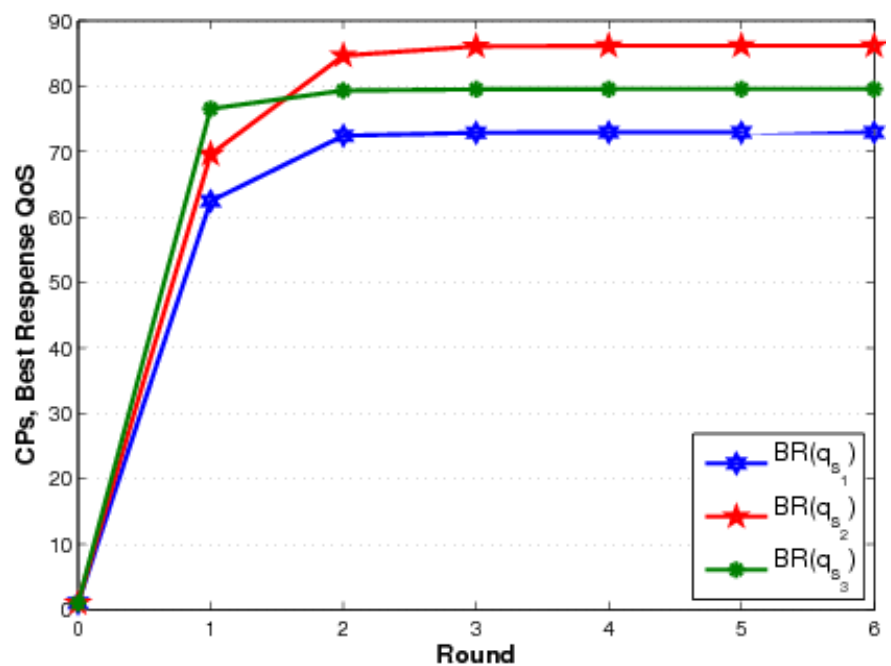

Figure 3. QoS Game: Convergence to the QoS Nash Equilibrium

Figures 2 and 3 present respectively curves of the convergence to Nash Equilibrium QoS and to Nash Equilibrium QoC. It is clear that the best response dynamics converges to the unique Nash equilibrium QoS and QoC. We also remark that the speed of convergence is relatively high. We also remark that the speed of convergence is relatively high. The gures 2 and 3 demonstrates the existence and uniqueness of a Nash equilibrium point at which no $\mathrm{CP}$ can profitably deviate given the strategies of another CP.

Second, we show the impact of the numbers of advertisers on the QoS and QoC of CPs. We vary the the numbers of advertisers for studying their impacts. 


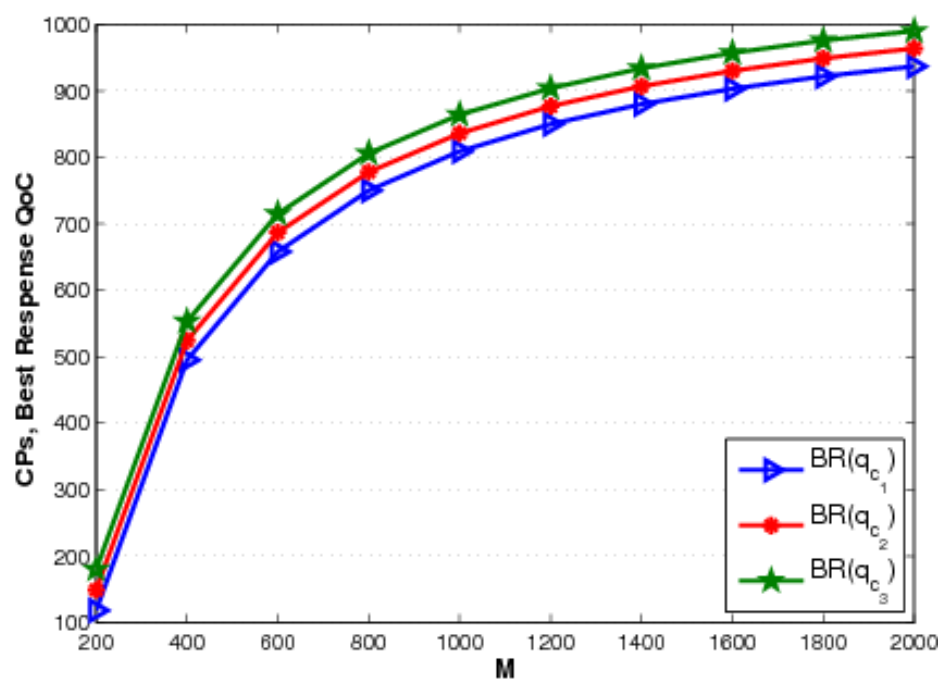

Figure 4. Equilibrium Price QoS as a Function of Number's Advertisers $M$

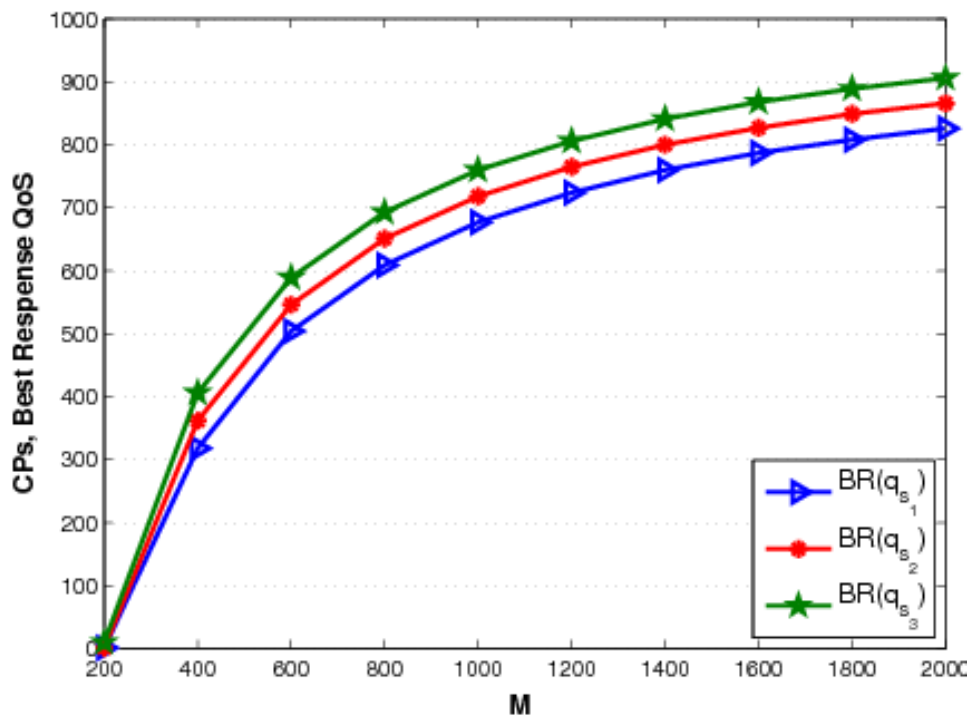

Figure 5. Equilibrium Price QoS as a Function of Number's Advertisers $M$

The impact of $M$ on QoS and QoC of the two CPs is illustrated in 4 and 5. Figure shows that QoC and QoS increase when $M$ become larger. The reason is that as $M$ increase, the revenue from advertisers increase too, which lead to increase the revenue of CPs. Therefore, the CPs invest for more to increase their QoS and QoC in order to induce increased demand from the end-users.

Third, we study the impact of the valuation $v_{i}$ on QoS and QoC of CPs by varying the valuation $v_{i}$. 


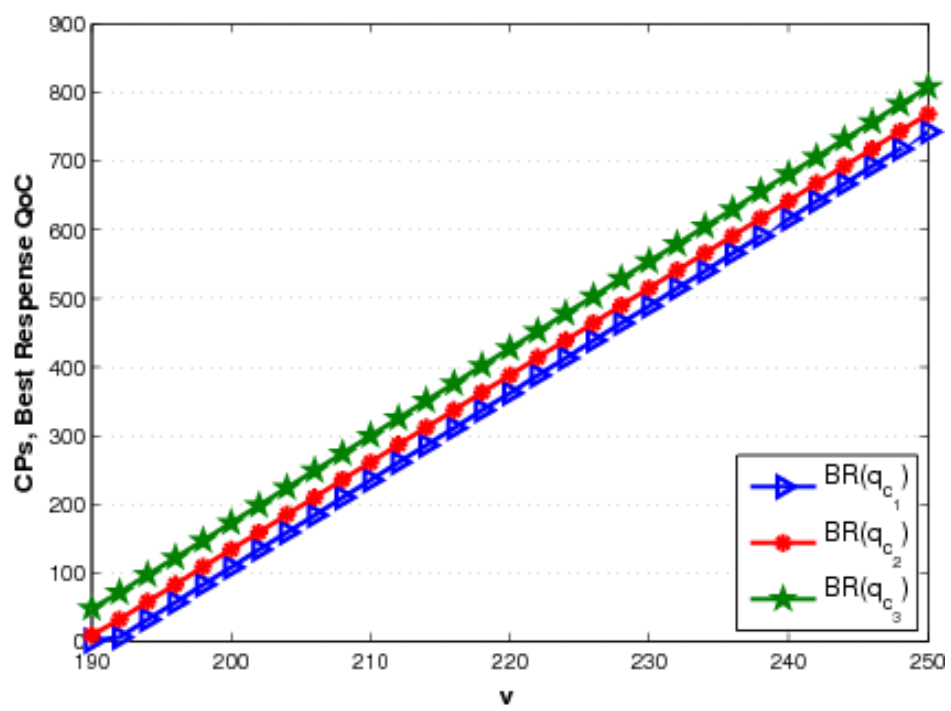

Figure 6. Equilibrium QoC as a Function of $\bar{v}$

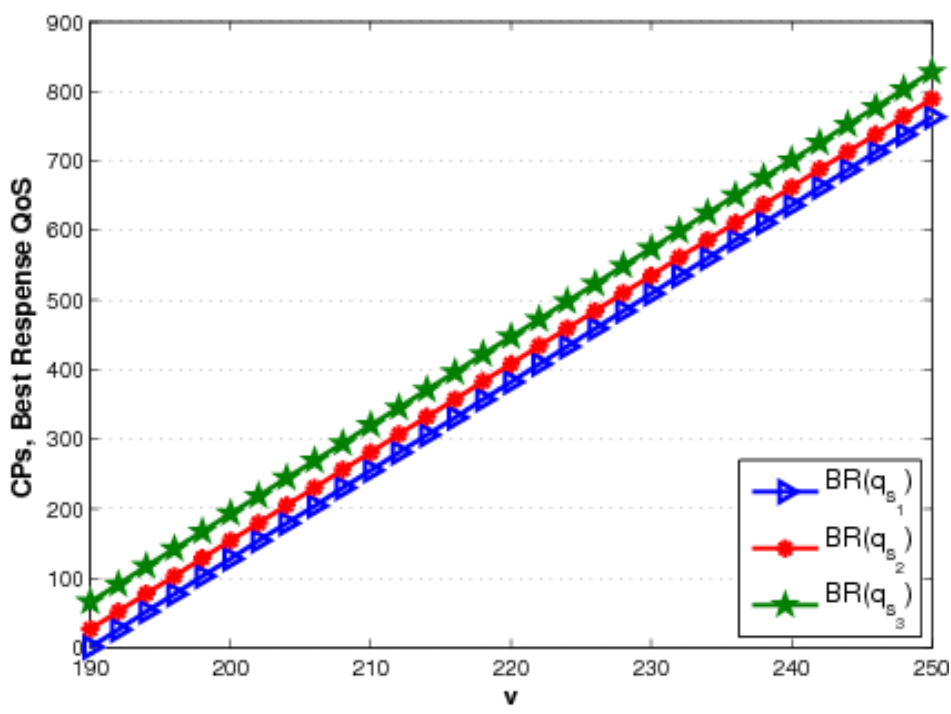

Figure 7. Equilibrium QoS as a Function of $\bar{v}$

We plot in Figures 6 and 7, respectively, the interplay of valuation $v$ on QoS and QoC at Nash equilibrium, for both CPs that we consider in this example. On the one hand, we note that the equilibrium QoS and QoC for both CPs is increasing with respect to the valuation $v$. When the valuation $v$ decided by the advertisers is cheaper, the revenue of CPs increases, then CPs invest to offer better QoS and QoC.

In the following, we discuss the impact of the system parameters on the system efficiency in terms of Price of anarchy: 


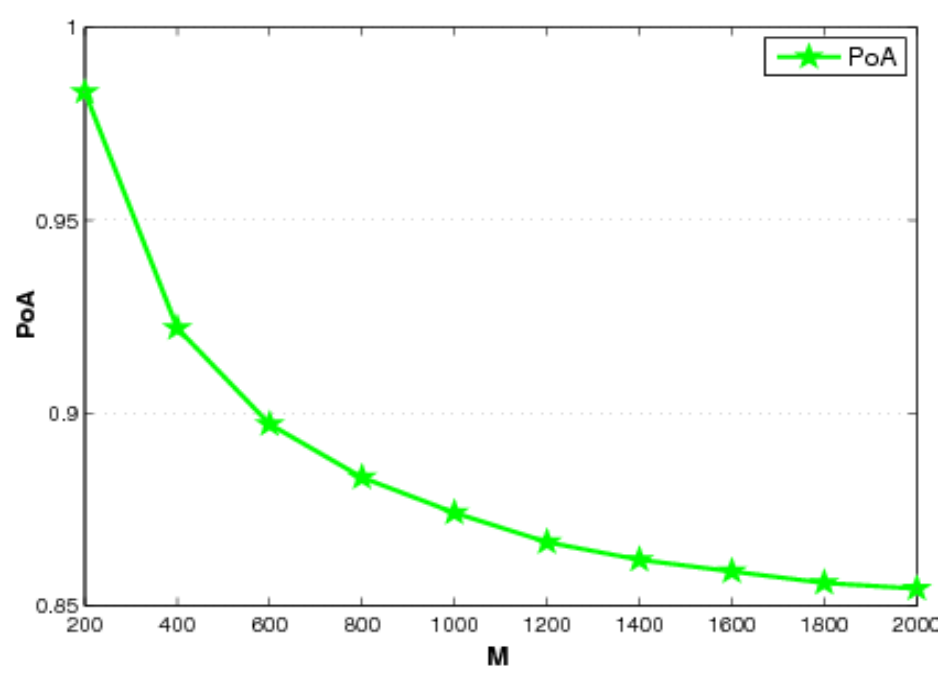

Figure 8. Price of Anarchy as a Function of Number's Advertisers $M$

Figure 8 shows the CPs PoA variation curve as a function of $M$ number of advertisers. The price of anarchy decreases with respect to $M$. When $M$ is lower the price of anarchy is socially efficient, moreover, when $M$ is lower the income from advertisers decrease and CPs cooperate with each other for optimizing the Nash equilibrium. On the other hand, when $M$ increase the price of anarchy is lower then the Nash equilibrium is not socially efficient, CPs are selfish and each one seeks to maximize its profit individually.

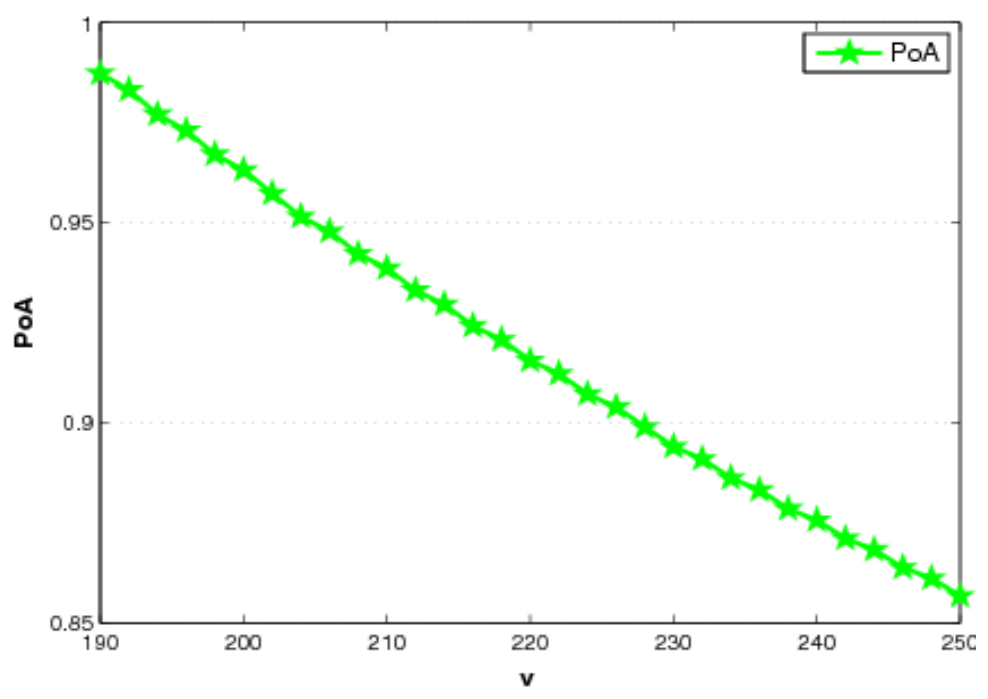

Figure 9. Price of Anarchy as a Function of $\bar{v}$

We plot the PoA variation curve of CPs as a function of valuation $v$ in Figure 9. In that figure, we first notice that the price of anarchy decreases when $v$ increases. When valuation $v$ is lower, the income from advertisers decrease and CPs cooperate with each other for optimizing the Nash equilibrium. On the other hand, when $v$ increase the price of anarchy is lower then the Nash equilibrium is not socially efficient, CPs are selfish and each one seeks to maximize its profit. 


\section{Conclusion}

Using fundamental concepts of game theory to analyze networked markets is not only useful from a theoretical perspective but it can also lead to practical contributions in network economics problems. In our work, we studied network economic model that includes end-users, content providers $(\mathrm{CP})$ and advertisers. We studied CPs obtains revenue from advertisers. We analyzed competition between $\mathrm{CP}$ as a function of two parameter QoS and QoC. On the other hand, we demonstrated the existence and uniqueness of the Nash equilibrium, then we applied the Best response Algorithm for learning Nash equilibrium. Also, we used the price of anarchy to evaluate the effectiveness of Nash equilibrium. We have shown also some results concerning the effect of numbers of advertisers, valuation on the CPs policies. The results found in this work show that CPs benefit form advertisers. We plan to extend our analysis to networks with several ISPs, CPs, advertisers and end-users.

\section{References}

[1] W. B. Norton and B. William, "The evolution of the US Internet peering ecosystem", Equinix white papers, (2004).

[2] A. Dhamdhere and C. Dovrolis, "Twelve years in the evolution of the internet ecosystem", IEEE/ACM Transactions on Networking (ToN), vol. 19, no. 5, (2011), pp. 1420-1433.

[3] Z. Han, "Game theory in wireless and communication networks: theory, models, and applications", Cambridge University Press, (2012).

[4] D. Ait Omar, M. Outanoute, M. Baslam, M. Fakir and B. Bouikhalne, "Joint Price and QoS Competition with Bounded Rational Customers", Networked Systems: 5th International Conference, NETYS 2017, Marrakech, Morocco, Proceedings, A. El Abbadi and B. Garbinato, Eds., Cham, (2017) May 17-19, pp. 457-471.

[5] H. Garmani, M. Baslam and M. Jourhmane, "Caching Games between ISP in Information Centric Network", International Journal of Control and Automation, vol. 11, no. 4, (2018), pp. 125-142.

[6] V. G. Douros, S. E. Elayoubi, E. Altman and Y. Hayel, "Caching games between Content Providers and Internet Service Providers", Performance Evaluation, vol. 113, (2017), pp. 13-25.

[7] M. Baslam, R. El-Azouzi, E. Sabir, L. Echabbi and E-H. Bouyakhf, "A Game Theoretic Analysis of Price-QoS Market Share in Presence of Adversarial Service Providers”, In Hanappi, Hardy, editors, Game Theory Relaunched, InTech, (2013).

[8] C. Zhang, B. Gu, Z. Liu, K. Yamori and Y. Tanaka, "A stackelberg game based analysis for interactions among Internet service provider, content provider, and advertisers", Consumer Communications \& Networking Conference (CCNC), 2017 14th IEEE Annual, (2017), pp. 231-235.

[9] P. Maillé and B. Tuffin, "Non-neutrality Pushed by Big Content Providers", International Conference on the Economics of Grids, Clouds, Systems, and Services, (2017), pp. 29-39.

[10] T. Jiménez, Y. Hayel and E. Altman, "Competition in access to content", Networking 2012, (2012), pp. 211-222.

[11] E. Altman, "In which content to specialize? a game theoretic analysis", International Conference on Research in Networking, (2012), pp. 121-125.

[12] N. Im, J. Mo and J. Park, "Revenue Sharing of ISP and CP in a Competitive Environment", International Conference on Game Theory for Networks, (2016), pp. 113-121.

[13] C. Zhang, B. Gu, K. Yamori, S. Xu and Y. Tanaka, "A novel Stackelberg-Bertrand game model for pricing content provider", Proceedings of the 8th International Conference on Mobile Multimedia Communications, (2015), pp. 128-132.

[14] P. Maillé and B. Tuffin, "Preventing competition using side payments: when non-neutrality creates barriers to entry", NETNOMICS: Economic Research and Electronic Networking, vol. 18, no. 1, (2017), pp. 3-22.

[15] M. K. Hanawal and E. Altman, "Network non-neutrality through preferential signaling", Modeling \& Optimization in Mobile, Ad Hoc \& Wireless Networks (WiOpt), 2013 11th International Symposium, (2013), pp. 232-239.

[16] S. Saberi, A. Nagurney and T. Wolf, "A network economic game theory model of a service-oriented Internet with price and quality competition in both content and network provision", Service Science, vol. 6, no. 4, (2014), pp. 229-250.

[17] R. El Azouzi, E. Altman and L. Wynter, "Telecommunications network equilibrium with price and quality-of-service characteristics", Edited J. Charzinski, R. Lehnert and P. Tran-Gia, Providing Quality of Service in Heterogeneous Environments Proceedings of the 18th International Teletraffic Congress-ITC-18 in Teletraffic Science and Engineering, Elsevier, (2003), pp. 369-378. 
[18] F. Bernstein and A. Federgruen, "A general equilibrium model for industries with price and service competition”, Operations research, vol. 52, no. 6, (2004), pp. 868-886.

[19] M. Baslam, L. Echabbi, R. El-Azouzi and E. Sabir, "Joint Price and QoS Market Share Game with Adversarial Service Providers and Migrating Customers", Game Theory for Networks, (2011), pp. 642-657.

[20] J. J. Liu and D. M. Chiu, "Mathematical modeling of competition in sponsored search market", Proceedings of the 2010 Workshop on Economics of Networks, Systems, and Computation, (2010), pp 10.

[21] S. Lasaulce, M. Debbah and E. Altman, "Methodologies for analyzing equilibria in wireless games", IEEE Signal Processing Magazine, vol. 26, no. 5, (2009), pp. 41-52.

[22] E. Koutsoupias and C. Papadimitriou, "Worst-case equilibria", Annual Symposium on Theoretical Aspects of Computer Science, (1999), pp. 404-413. 